\title{
Usefulness of keystone island flap for circumferential defect around a urostomy
}

\author{
Atsumori Hamahata Takashi Saitou • \\ Masashi Ishikawa $\cdot$ Noriko Kubota $\cdot$ \\ Takashi Yamaki $\cdot$ Hiroyuki Sakurai
}

Received: 8 December 2012 / Accepted: 22 January 2013/Published online: 7 February 2013

(C) The Japan Society of Clinical Oncology 2013

\begin{abstract}
Free perforator flap, perforator propeller flap, and super microsurgery flap are newly used to cover skin defects in all parts of the body. However, these methods require complex surgical techniques, long operation times, and carry the risk of unstable flap circulation. Recently, the keystone island flap has been reported as increasingly being used to cover skin defects. The elevation of the keystone island flap is easier compared to other flaps, and flap circulation is very stable. It has been reported that the keystone flap was successfully used in surgeries of the head and neck, trunk, and extremities. Meanwhile, there are few reports about reconstructive methods used for the circumferential defect around abdominal stomas. In this report we describe a patient who had an abdominal circumferential skin defect surrounding a urostomy caused by tumor resection. The defect was successfully reconstructed using the keystone island flap method.
\end{abstract}

Keywords Keystone island flap - Abdominal stoma . Extramammary Paget disease $\cdot$ Circumferential defect

\footnotetext{
A. Hamahata $(\bowtie) \cdot T$. Saitou

Department of Plastic and Reconstructive Surgery,

Saitama Cancer Center, 818 Komuro Inamachi,

Kitaadachigunn, Saitama, Japan

e-mail: a.hamahata@gmail.com

M. Ishikawa $\cdot$ N. Kubota

Department of Dermatology, Saitama Cancer Center,

Kitaadachigunn, Japan

T. Yamaki · H. Sakurai

Department of Plastic and Reconstructive Surgery,

Tokyo Women's Medical University, Shinjukuku, Japan
}

\section{Introduction}

Free perforator flap and perforator propeller flap have been most commonly used for abdominal skin defects. More recently, perforator propeller flaps based on the deep inferior epigastric perforator or island anterior intercostal artery perforator have been reported as reconstructive methods for abdominal skin defects. However, complex operative techniques and long operation times are required, and the perforator propeller flap carries the risk of flap congestion due to kinking and twisting of the pedicle. In terms of alternative methods, the keystone island flap was described by Behan in 2003 [1]. There are several reports that the keystone flap has been successfully used in surgeries involving the neck, trunk, and extremities. Meanwhile, reconstructive methods describing the circumferential defect surrounding abdominal stomas are scarce. Thus, this case report describes a reconstructive method using the keystone island flap for a circumferential skin defect surrounding a urostomy.

\section{Case report}

A 73-year-old man was referred to us presenting with a skin tumor surrounding his urostomy. He had received total pelvic exenteration 14 years previously due to rectal carcinoma, and had a urostomy on the right side of his abdominal wall and a colostomy on the wall's left side. Following dermatological biopsy, extramammary Paget disease was diagnosed (Fig. 1). No swelling of his groin area lymph nodes was observed.

The urinary catheter was inserted in the urostomy and the mucosa of the urostomy was temporarily closed by pursestring suture to prevent urine from escaping the stoma. The 


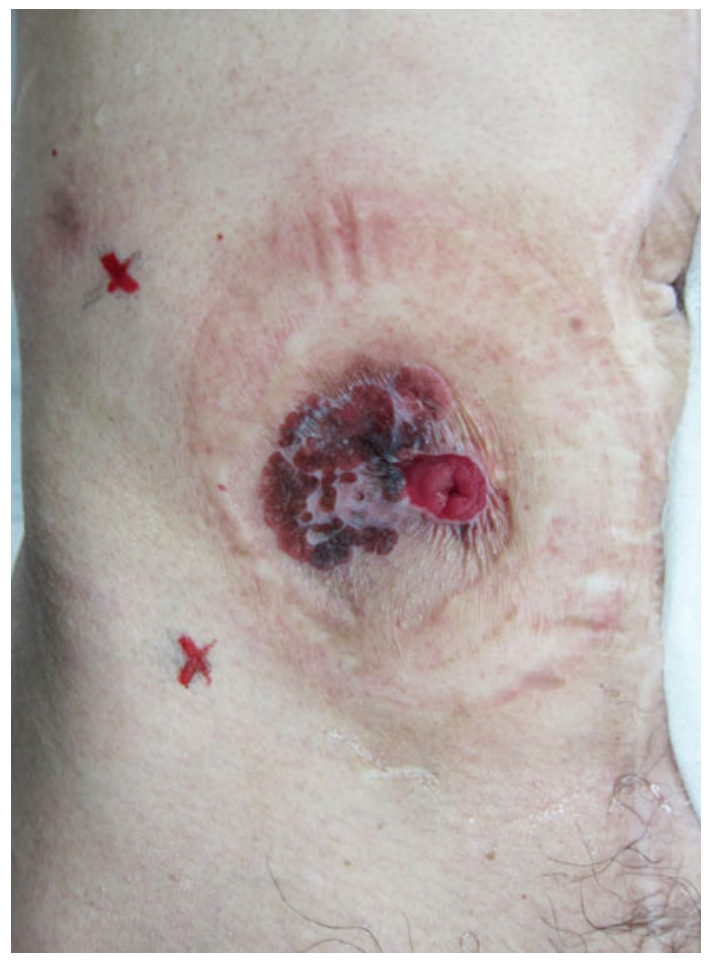

Fig. 1 The patient had extramammary Paget disease around the urostomy. He had a colostomy on the left side of his abdominal wall

tumor lesion around his urostomy was excised under general anesthesia by dermatologists. A portion of mucosa near the tumor was excised for surgical margin. The skin defect was a circumferential defect surrounding the urostomy (Fig. 2). The skin defect size was $9 \mathrm{~cm}$ in diameter including the stoma. The defect's lateral side was about $5 \mathrm{~cm}$ in length and the medial side was about $2 \mathrm{~cm}$ in length. The keystone island flap was designed as shown in Fig. 2. As described by Khouri et al. [2] the movable flap width $(5 \mathrm{~cm})$ was equal to the defect width $(5 \mathrm{~cm}$; lateral skin margin to stoma). The total flap size was $11 \times 24 \mathrm{~cm}$. The flap was elevated over the fascia and moved medially. The medial side defect could be closed directly only by dissecting the left side of the skin (Fig. 3). The subdermal layer of the flap and the seromuscular layer of the stoma were sutured with 3-0 vicryl, and the flap skin and mucosa were sutured with 4-0 vicryl. Suction drains were placed under the lateral side of the flap. A stoma pouch was fitted to the stoma immediately following the operation. The operation time was $1 \mathrm{~h} 51 \mathrm{~min}$, and the blood loss was $132 \mathrm{~g}$.

The stoma pouch was changed once daily from postoperative day 1 to day 3 , and the stoma's mucosa was observed for changes in color. The patient was discharged from the hospital on postoperative day 7 without any complications such as infection, stoma necrosis, and flap necrosis. The patient's abdominal flap and urostomy exhibited good outcomes, with no sign of tumor recurrence or complications after 6 months (Fig. 4).

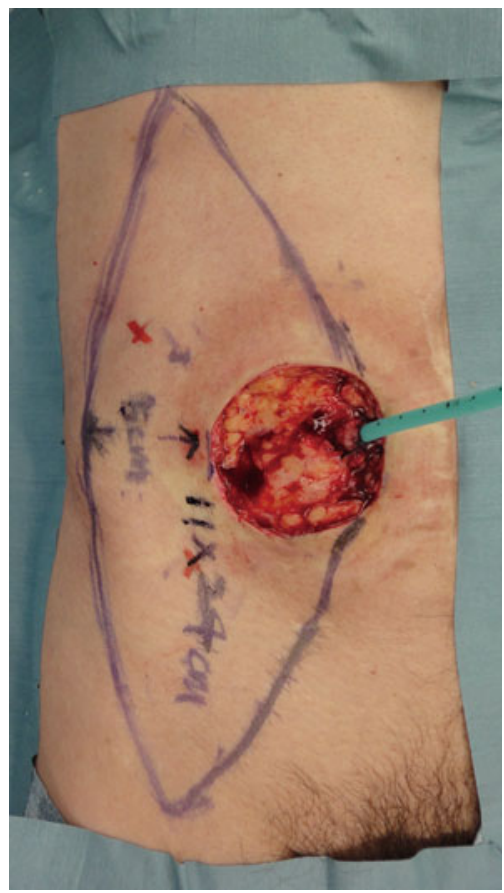

Fig. 2 The skin defect was $9 \mathrm{~cm}$ in diameter. The lateral defect to the stoma was $5 \mathrm{~cm}$ wide and the medial defect to the stoma was $2 \mathrm{~cm}$ wide. The flap design was in total $11 \times 24 \mathrm{~cm}$ and the flap width just beside the defect was $5 \mathrm{~cm}$ wide
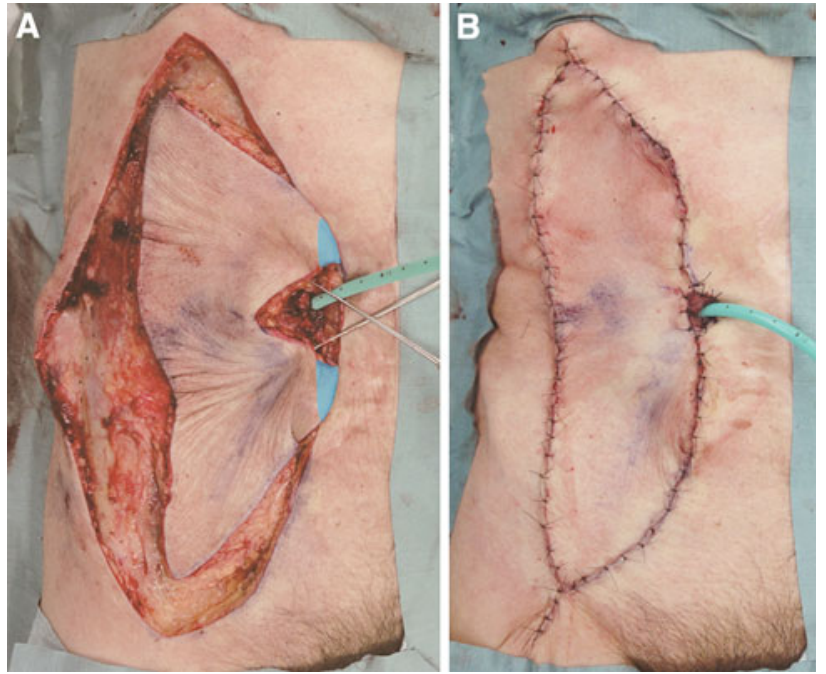

Fig. 3 The flap was elevated and the flap tip (blue colored area) was trimmed. The medial side of the skin was also dissected (a). The circumferential skin defect around the urostomy was completely closed (b)

\section{Discussion}

The free perforator flap, perforator propeller flap, and super microsurgery flap have been popular flaps for use in plastic surgery. However, use of these flaps requires long operation times and result in unstable flap circulation, especially for inexperienced surgical teams. Therefore, use of a 


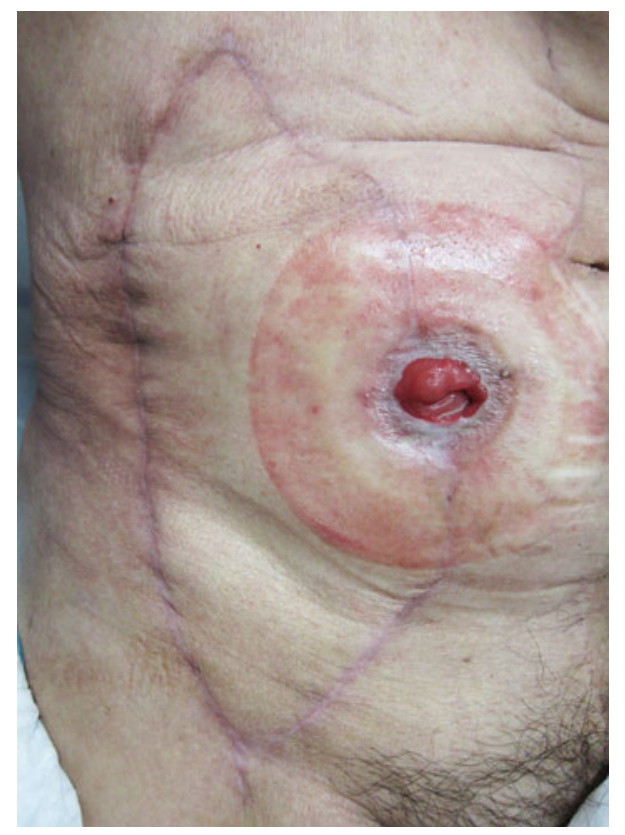

Fig. 4 After 6 months the patient had returned to normal life and the urostomy pouch could be changed without any trouble

different, more robust flap is warranted for delicate situations such as operations on elderly patients or on patients with severe comorbidities. The keystone island flap was first reported by Behan in 2003, and the flap has gained in worldwide popularity since that time [1]. The first anatomical studies of the flap were reported by Pelissier et al. [3] in 2007. There are several modifications of the flap, with its use concentrated to neck, trunk, and extremity areas [4]. The flap is easy to elevate and has a very stable vascular circulation. We used this stable keystone flap for the skin defect surrounding a urostomy. There have been no definitive reports about reconstruction of the circumferential skin defects surrounding the stoma, although Ang et al.'s [5] report indicated that the pedicled 'propeller' deep inferior epigastric perforator (DIEP) flap was used near a stoma. For skin surrounding a stoma, a robust and well-vascularized flap is needed because the border of skin and stoma is easily infected and the skin surrounding a stoma is continuously contaminated with urine. In our case, although the patent had a colostomy in his left-side abdominal wall, the medial side of the skin defect (about $2 \mathrm{~cm}$ wide) could be closed directly. For the lateral side of the skin defect, the robust and stable keystone island flap was used. The design of the flap was guided by Khouri et al.'s [2] study, and the flap width adjacent to the defect was $5 \mathrm{~cm}$. The total flap size was $11 \times 24 \mathrm{~cm}$. The flap was trimmed and the skin defect could then be closed easily. Even if the medial side of the skin defect had been wider, the skin defect could have been closed using the trimmed skin tissue of the keystone island flap. If the patient did not have a colostomy and the medial skin defect were much larger, another keystone island flap could have been elevated on the opposite side of the abdominal wall.

The keystone island flap is less susceptible to flap congestion because the flap only slides skin tissue on perforators; conversely, the perforator propeller flap has a risk of flap congestion because the perforator pedicle twists and kinks after turning the flap. Moreover, the operation time is shorter because the keystone island flap does not require intricate pedicle dissection. However, a recommended limitation of this method is that the defect size should be small to moderate, and the regional skin should also remain intact. In cases of large abdominal wall defect, some additional free flaps would be necessary.

The free perforator flap, perforator propeller flap, and super microsurgery flap are excellent techniques and remain warranted in some situations. However, if similar results can be obtained using alternate operative options, the method with the least risk of complications is preferred, especially for elderly patients and patents with comorbidities. The patient in this study was elderly and had a respiratory disorder. He could not tolerate lengthy operation times nor a large loss of blood. Therefore, we chose the keystone flap technique for this patent and successfully reconstructed the circumferential skin defect around the stoma.

Acknowledgments We thank Jeffrey D. Meserve for his editorial assistance.

Conflict of interest The authors declare that they have no conflict of interest.

\section{References}

1. Behan FC (2003) The keystone design perforator island flap in reconstructive surgery. ANZ J Surg 73(3):112-120

2. Khouri JS, Egeland BM, Daily SD et al (2011) The keystone island flap: use in large defects of the trunk and extremities in soft-tissue reconstruction. Plast Reconstr Surg 127(3):1212-1221

3. Pelissier P, Santoul M, Pinsolle V et al (2007) The keystone design perforator island flap. Part I: anatomic study. J Plast Reconstr Aesthet Surg 60(8):883-887

4. Behan F, Sizeland A, Gilmour F et al (2010) Use of the keystone island flap for advanced head and neck cancer in the elderly-a principle of amelioration. J Plast Reconstr Aesthet Surg 63(5):739-745

5. Ang GG, Rozen WM, Chauhan A, Acosta R (2011) The pedicled 'propeller' deep inferior epigastric perforator (DIEP) flap for a large abdominal wall defect. J Plast Reconstr Aesthet Surg 64(1):133-135 